\title{
Constrained Simultaneous and Near-Simultaneous Embeddings
}

\author{
Fabrizio Frati ${ }^{1}$, Michael Kaufmann ${ }^{2}$, and Stephen G. Kobourov ${ }^{3}$ \\ ${ }^{1}$ Dipartimento di Informatica e Automazione - Università Roma Tre, Italy \\ ${ }^{2}$ Wilhelm-Schickard-Institut für Informatik - Universität Tübingen, Germany \\ ${ }^{3}$ Department of Computer Science - University of Arizona, U.S.A.
}

\begin{abstract}
A geometric simultaneous embedding of two graphs $G_{1}=$ $\left(V_{1}, E_{1}\right)$ and $G_{2}=\left(V_{2}, E_{2}\right)$ with a bijective mapping of their vertex sets $\gamma: V_{1} \rightarrow V_{2}$ is a pair of planar straight-line drawings $\Gamma_{1}$ of $G_{1}$ and $\Gamma_{2}$ of $G_{2}$, such that each vertex $v_{2}=\gamma\left(v_{1}\right)$ is mapped in $\Gamma_{2}$ to the same point where $v_{1}$ is mapped in $\Gamma_{1}$, where $v_{1} \in V_{1}$ and $v_{2} \in V_{2}$.

In this paper we examine several constrained versions and a relaxed version of the geometric simultaneous embedding problem. We show that if the input graphs are assumed to share no common edges this does not seem to yield large classes of graphs that can be simultaneously embedded. Further, if a prescribed combinatorial embedding for each input graph must be preserved, then we can answer some of the problems that are still open for geometric simultaneous embedding. Finally, we present some positive and negative results on the near-simultaneous embedding problem, in which vertices are not mapped exactly to the same but to "near" points in the different drawings.
\end{abstract}

\section{Introduction}

Graph drawing techniques are commonly used to visualize relationships between objects, where the objects are the vertices of the graph and the relationships are captured by the edges in the graph. Simultaneous embedding is a problem that arises when visualizing two or more relationships defined on the same set of objects. If the graphs corresponding to these relationships are planar, the aim of simultaneous embedding is to find point locations in the plane for the vertices of the graphs, so that each of the graphs can be realized on the same point-set without edge crossings. To ensure good readability of the drawings, it is preferable if the edges are drawn as straight-line segments. This problem is known as geometric simultaneous embedding. It has been shown that only a few classes of graphs can be embedded simultaneously with straight-line segments. Brass et al. [1, Erten and Kobourov [5], and Geyer et al. [8] showed that three paths, a planar graph and a path, and two trees do not admit geometric simultaneous embeddings. On the positive side, an algorithm for geometric simultaneous embedding of two caterpillars [1] is the strongest known result.

As geometric simultaneous embedding turns out to be very restrictive, it is natural to relax some of the constraints of the problem. Not insisting on straightline edges led to positive results such as a linear-time algorithm by Erten and

S.-H. Hong, T. Nishizeki, and W. Quan (Eds.): GD 2007, LNCS 4875, pp. 268-279, 2007.

(C) Springer-Verlag Berlin Heidelberg 2007 
Table 1. Known results and our contribution on geometric simultaneous embedding (Geometric), geometric simultaneous embedding with no common edges (Disj. Edges), geometric simultaneous drawing with fixed embedding (Fixed Embedding), geometric simultaneous drawing with fixed embedding and no common edges (Disj. Edges, Fixed Embedding).

\begin{tabular}{|c|c|c|c|c|}
\hline & Geometric & Disj. Edges & Fixed Emb. & Disj. Edges, Fixed Emb. \\
\hline path + path & \begin{tabular}{|l|l|} 
YES & 1 \\
\end{tabular} & YES 1 & YES 1 & \begin{tabular}{l|l} 
YES & 1 \\
\end{tabular} \\
\hline star + path & YES 1 ] & YES 1 & $\begin{array}{l}\text { YES Sec. } 4.1 \\
4.1\end{array}$ & YES Sec. 4.1 \\
\hline double-star + path & \begin{tabular}{l|l} 
YES 1 \\
\end{tabular} & $\begin{array}{lll}\text { YES } 11 \\
\end{array}$ & $?$ & YES Sec. 4.1 \\
\hline caterpillar + path & \begin{tabular}{ll|} 
YES 1$]$ \\
\end{tabular} & \begin{tabular}{l|l|} 
YES 1 \\
\end{tabular} & $?$ & $?$ \\
\hline caterpillar + caterpillar & YES 1] & YES 1] & NO Sec. 4.2 & NO Sec. 4.2 \\
\hline 3 paths & $\mathrm{NO}[1]$ & $?$ & $\mathrm{NO}[1]$ & $?$ \\
\hline tree + path & $?$ & $?$ & $?$ & $?$ \\
\hline tree + cycle & $?$ & $?$ & $?$ & $?$ \\
\hline tree + caterpillar & $?$ & $?$ & NO Sec. 4.2 & NO Sec. 4.2 \\
\hline outerplanar + path & $?$ & $?$ & NO Sec. 4.3 & NO Sec. 4.3 \\
\hline outerplanar + caterpillar & $?$ & $?$ & NO Sec. 4.2 & NO Sec. 4.2 \\
\hline outerplanar + cycle & $?$ & $?$ & NO Sec. 4.3 & NO Sec. 4.3 \\
\hline tree + tree & NO 8 & $?$ & NO 8 & NO Sec. 4.2 \\
\hline outerplanar + tree & \begin{tabular}{l|l|} 
NO & 8 \\
\end{tabular} & $?$ & $\begin{array}{ll}\mathrm{NO} & 8 \\
\end{array}$ & NO Sec. 4.2 \\
\hline outerplanar + outerplanar & NO [1] & $?$ & NO [1] & NO Sec. 4.2 \\
\hline planar + path & NO $[5$ & NO Sec. 3 & NO [5] & NO Sec. 3 \\
\hline planar + tree & \begin{tabular}{l|l|} 
NO & 5 \\
\end{tabular} & NO Sec. 3 & $\begin{array}{ll}\mathrm{NO} & 5 \\
\end{array}$ & NO Sec. 3 \\
\hline planar + planar & NO 5 & NO Sec. 3 & NO 5 & NO Sec. 3 \\
\hline
\end{tabular}

Kobourov for embedding any pair of planar graphs with at most three bends per edge, or any pair of trees with at most two bends per edge [5]. In such results it is allowed for an edge connecting a pair of vertices to be represented by different Jordan curves in different drawings. As this can be detrimental to the readability of the drawings, several papers considered a slightly more constrained version of this problem, namely, simultaneous embedding with fixed edges, in which bends are allowed, however, an edge connecting the same pair of vertices must be drawn in exactly the same way in all drawings. Di Giacomo and Liotta [4] showed that outerplanar graphs can be simultaneously embedded with fixed edges with paths or cycles using at most one bend per edge. Frati $[6$ showed that a planar graph and a tree can also be simultaneously embedded with fixed edges.

Studying the existing variants of simultaneous embedding led to practical embedding algorithms for some graph classes and techniques for simultaneous embedding have been used in visualizing evolving and dynamic graphs [2]. However, many problems remain theoretically open and in practice algorithms applying these ideas to evolving and dynamic graphs do not provide any guarantees on the quality of the resulting layouts. With this in mind, we consider three further variants of the geometric simultaneous embedding problem.

Most of the proofs about the non-existence of simultaneous embeddings exploit the presence of common edges between the input graphs. Hence, it is natural ask if larger classes of graphs have geometric simultaneous embeddings when no edges are shared. In Section 3 we answer in the negative for planar graph-path pairs, generalizing the result in [5], where it is shown that a planar graph and a path that share edges do not admit a geometric simultaneous embedding.

In Section 4 we consider the problem of geometric simultaneous embedding in which the embeddings for the graphs are fixed. We call this setting geometric simultaneous embedding with fixed embeddings. Clearly, negative results known 
for geometric simultaneous embedding remain valid here. We show that some classes of graphs that have geometric simultaneous embeddings do not admit one with individually fixed embeddings. In particular, we prove such a negative result for caterpillar-caterpillar pairs. Moreover, in the fixed embedding setting we are able to solve problems that are still open for geometric simultaneous embedding. Namely, we provide an outerplanar-path pair that has no geometric simultaneous drawing with fixed embedding. All the negative results claimed are still valid if the input graphs are assumed to not share edges. On the other hand, we partially cover the known positive results for geometric simultaneous embedding, by showing that a star and a path can always be realized and that a double-star and a path can always be realized if they do not share edges.

In the quest for more practical setting where we can guarantee some properties of the layouts, in Section 5 we study a variant we call geometric near-simultaneous embedding. In this setting edges are straight lines and vertices representing the same entity in different graphs can be placed not exactly in the same point but just in "near" points. Assuming vertices are placed on the grid, we show that there exist pairs of $n$-vertex planar graphs in which vertices that represent the same entity in different graphs must be placed at distance $\Omega(n)$. We then consider graphs "similar" in their combinatorial structure, describing algorithms which guarantee that vertices representing the same entity have only constant displacement from one drawing to the next. Such algorithms can be used to guarantee limited displacement in dynamic graph drawings.

Due to space limitations, we leave out some proofs, that can be found in [7].

\section{Preliminaries}

We summarize basic terminology used in this paper; for more details see [311. A straight-line drawing of a graph is a mapping of each vertex to a unique point in the plane and of each edge to a segment between the endpoints of the edge. A planar drawing is one in which no two edges intersect. A planar graph is a graph that admits a planar drawing. A grid drawing is one in which every vertex is placed at a point with integer coordinates in the plane. An embedding of a graph is a circular ordering of the edges incident on each vertex of $G$. An embedding of a graph specifies the faces in any drawing respecting such an embedding, even though the embedding does not determine which one is the external face. A graph is triconnected if for every pair of distinct vertices there exist three vertex-disjoint paths connecting them. A triconnected graph has an unique embedding, up to a reversal of its adjacency lists.

An outerplanar graph is a graph that admits a drawing in which all the vertices are incident to the same face. A caterpillar is a tree in which the removal of all the leaves and their incident edges yields a path. A star (double-star) is a caterpillar with only one vertex (two vertices) of degree greater than one.

A geometric simultaneous embedding of two graphs $G_{1}=\left(V_{1}, E_{1}\right)$ and $G_{2}=$ $\left(V_{2}, E_{2}\right)$ with a bijective mapping $\gamma$ of their vertex sets is a pair of planar 
straight-line drawings $\Gamma_{1}$ of $G_{1}$ and $\Gamma_{2}$ of $G_{2}$, such that each vertex $v_{2}=\gamma\left(v_{1}\right)$ is mapped in $\Gamma_{2}$ to the same point where $v_{1}$ is mapped in $\Gamma_{1}$, where $v_{1} \in V_{1}$ and $v_{2} \in V_{2}$.

\section{Simultaneous Embedding without Common Edges}

We consider the geometric simultaneous embedding of graphs not sharing common edges, exhibiting a planar graph and a path that cannot be drawn simultaneously. We revisit the problem of embedding simultaneously graphs not sharing edges in the conclusions (Section 6).

Let $G^{*}$ be the planar graph on vertices $v_{1}, v_{2}, \ldots, v_{9}$ shown in Fig. 11(a). Since $G^{*}$ is triconnected, it has the same faces in any planar embedding. Let $F^{*}$ denote the triangular face $\Delta v_{1} v_{3} v_{9}$ and $P^{*}$ be the path $\left(v_{1}, v_{2}, v_{3}, v_{4}, v_{5}, v_{6}, v_{7}, v_{8}, v_{9}\right)$.

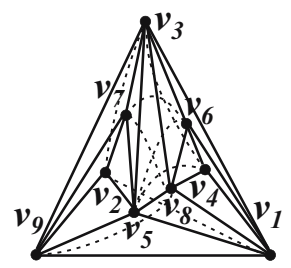

(a)

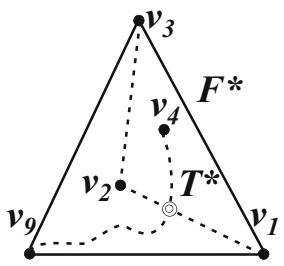

(b)

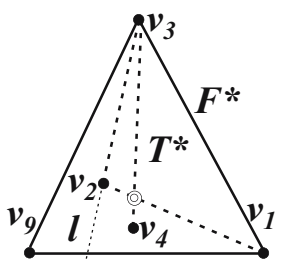

(c)

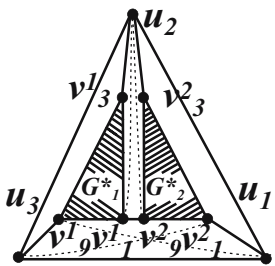

(d)

Fig. 1. (a) Planar graph $G^{*}$ drawn with solid edges and path $P^{*}$ drawn with dashed edges; (b)-(c) Illustrations for the proof of Lemma 1 (d) Planar graph $G$ drawn with solid edges and path $P$ drawn with dashed edges.

Lemma 1. There does not exist a geometric simultaneous embedding of $G^{*}$ and $P^{*}$ in which the external face of $G^{*}$ is $F^{*}$.

Proof: All vertices of $G^{*}$, other than $v_{1}, v_{3}$ and $v_{9}$, are inside $F^{*}$ as $F^{*}$ is the external face of $G^{*}$. Consider the triangle $T^{*}$ formed by edges $\left(v_{1}, v_{2}\right),\left(v_{2}, v_{3}\right)$ of $P^{*}$, and by edge $\left(v_{1}, v_{3}\right)$ of $G^{*}$. Since $v_{9}$ is incident to $F^{*}$, it must lie outside $T^{*}$. Let $l$ be the line passing through $v_{2}$ and $v_{3} ; l$ separates the plane in two open halfplanes, one containing $v_{9}$, called the exterior part of $l$, and one not containing $v_{9}$, called the interior part of $l$. Consider the possible placements of $v_{4}$. If $v_{4}$ is placed inside $T^{*}$ then the subpath of $P^{*}$ composed of edges $\left(v_{1}, v_{2}\right)$ and $\left(v_{2}, v_{3}\right)$ crosses the subpath of $P^{*}$ connecting $v_{4}$, that lies inside $T^{*}$, and $v_{9}$, that lies outside $T^{*}$; see Fig. 1(b). Suppose $v_{4}$ is placed outside $T^{*}$. Since vertex $v_{4}$ (vertex $v_{2}$ ) must lie inside triangle $\Delta v_{1} v_{3} v_{5}$ (inside triangle $\Delta v_{3} v_{5} v_{9}$ ), the clockwise order of edges $\left(v_{3}, v_{1}\right),\left(v_{3}, v_{5}\right),\left(v_{3}, v_{9}\right)$ of $G^{*}$ and edges $\left(v_{3}, v_{4}\right),\left(v_{3}, v_{2}\right)$ of $P^{*}$ around $v_{3}$ must be $\left(v_{3}, v_{1}\right),\left(v_{3}, v_{4}\right),\left(v_{3}, v_{5}\right),\left(v_{3}, v_{2}\right),\left(v_{3}, v_{9}\right)$. Therefore $v_{4}$ is in the interior part of $l$ and hence edge $\left(v_{1}, v_{2}\right)$ crosses edge $\left(v_{3}, v_{4}\right)$ in $P^{*}$; see Fig. 1(c).

Theorem 1. There exist a planar graph $G$, a path $P$, and a mapping between their vertices such that: (i) $G$ and $P$ do not share edges, and (ii) $G$ and $P$ have no geometric simultaneous embedding. 
Proof: We construct $G$ and $P$ out of two copies of $G^{*}$ and $P^{*}$ described above. Let $G_{1}^{*}$ and $G_{2}^{*}$ be two copies of $G^{*}$. Denote by $v_{i}^{j}$ the vertex of $G_{j}^{*}$ that corresponds to the vertex $v_{i}$ in $G^{*}$, where $j=1,2$ and $i=1, \ldots, 9$. Let $G$ be the graph composed of $G_{1}^{*}$ and $G_{2}^{*}$ together with three additional vertices $u_{1}, u_{2}$, and $u_{3}$ and eight additional edges $\left(u_{1}, u_{2}\right),\left(u_{1}, u_{3}\right),\left(u_{2}, u_{3}\right),\left(u_{1}, v_{1}^{2}\right),\left(u_{2}, v_{3}^{1}\right),\left(u_{2}, v_{3}^{2}\right)$, $\left(u_{3}, v_{9}^{1}\right)$, and $\left(v_{1}^{1}, v_{9}^{2}\right)$; see Fig. 11(d). Graph $G$ is triconnected and therefore it has exactly one planar embedding and it has the same faces in any plane drawing. Let $P$ be the path $\left(u_{1}, v_{9}^{1}, v_{8}^{1}, v_{7}^{1}, v_{6}^{1}, v_{5}^{1}, v_{4}^{1}, v_{3}^{1}, v_{2}^{1}, v_{1}^{1}, u_{2}, v_{9}^{2}, v_{8}^{2}, v_{7}^{2}, v_{6}^{2}, v_{5}^{2}, v_{4}^{2}, v_{3}^{2}, v_{2}^{2}\right.$, $\left.v_{1}^{2}, u_{3}\right)$. It is easy to verify that $G$ and $P$ do not share edges. Note that the subpaths of $P$ induced by the vertices of $G_{1}^{*}$ and by the vertices of $G_{2}^{*}$ play the same role that path $P^{*}$ plays for graph $G^{*}$ in Lemma 1 .

Let $F_{1}^{*}$ and $F_{2}^{*}$ denote cycles $\left(v_{1}^{1}, v_{3}^{1}, v_{9}^{1}\right)$ and $\left(v_{1}^{2}, v_{3}^{2}, v_{9}^{2}\right)$; these cycles are faces of $G_{1}^{*}$ and $G_{2}^{*}$. We now show that every plane drawing $\Gamma$ of $G$ determines a non-planar drawing of $P$. Consider the embedding $\mathcal{E}_{G}$ of $G$ obtained by choosing $\Delta u_{1} u_{2} u_{3}$ as external face; see Fig. 1(d). Choosing any face external to $F_{1}^{*}\left(F_{2}^{*}\right)$ in $\mathcal{E}_{G}$ as external face of $\Gamma$ leaves $G_{1}^{*}\left(G_{2}^{*}\right)$ embedded with external face $F_{1}^{*}$ $\left(F_{2}^{*}\right)$. Hence, we can apply Lemma 1 and conclude that there does not exist a simultaneous embedding of $G$ and $P$.

\section{Simultaneous Drawing with Fixed Embedding}

Next, we examine the possibility of embedding graphs simultaneously with straight-line edges and with fixed embeddings.

\subsection{Simultaneous Drawing of Stars, Double-Stars and Paths with Fixed Embedding}

Let $P$ be an $n$-vertex path and let $S$ be an $n$-vertex star with center $c$ and embedding $\mathcal{E}$. Let $P=\left(a_{1}, a_{2}, \ldots, a_{l}, c, b_{1}, b_{2}, \ldots, b_{m}\right)$, where one among sequences $\left(a_{1}, a_{2}, \ldots, a_{l}\right)$ and $\left(b_{1}, b_{2}, \ldots, b_{m}\right)$ could be empty. Draw $S$ with $c$ as leftmost point and with all edges in an order around $c$ consistent with $\mathcal{E}$, so that edge $\left(c, b_{1}\right)$, if it exists, is the uppermost edge of $S$. This can be done so that the $x$-coordinate of a vertex $b_{i}$ is greater than the one of a vertex $a_{j}$, with $1 \leq i \leq m$ and $1 \leq j \leq l$, the $x$-coordinate of a vertex $b_{i}$ is greater than the one of a vertex $b_{j}$, with $1 \leq j<i \leq m$, and the $x$-coordinate of a vertex $a_{i}$ is greater than the one of a vertex $a_{j}$, with $1 \leq i<j \leq l$; see Fig. 2(a). The resulting drawing of $S$ is clearly planar. Further, $P$ is not self-intersecting as it is realized by two $x$-monotone curves joined by an edge that is higher than every other edge of $P$. This yields the following result:

Theorem 2. An n-vertex star and an n-vertex path admit a geometric simultaneous embedding in which the star has a fixed prescribed embedding.

Now let $P$ be an $n$-vertex path and let $D$ be an $n$-vertex double-star with centers $c_{1}$ and $c_{2}$ and with embedding $\mathcal{E}$. Suppose $D$ and $P$ do not share edges. Let $P=\left(a_{1}, a_{2}, \ldots, a_{l}, c_{1}, b_{1}, b_{2}, \ldots, b_{m}, c_{2}, d_{1}, d_{2}, \ldots, d_{p}\right)$. Sequences $\left(a_{1}, a_{2}, \ldots, a_{l}\right)$ 


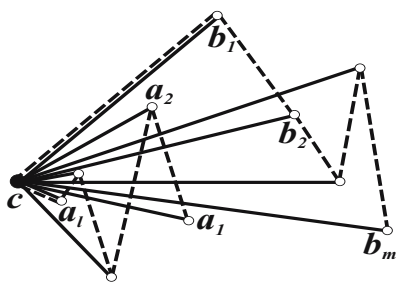

(a)

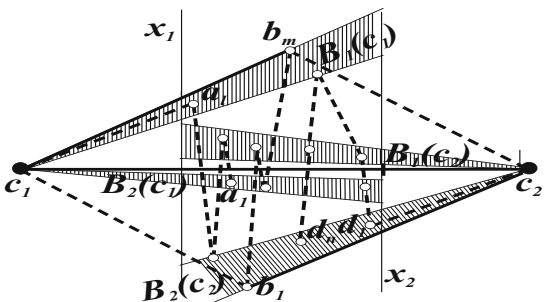

(b)

Fig. 2. (a) Simultaneous embedding of a star and a path; (b) Simultaneous embedding of a double-star and a path not sharing edges

and $\left(d_{1}, d_{2}, \ldots, d_{p}\right)$ could be empty, while $m \geq 2$. Further, $b_{1}$ is neighbor of $c_{2}$ and $b_{m}$ is neighbor of $c_{1}$ in $D$; see Fig. 2(b). Group the edges incident to $c_{1}$ (incident to $c_{2}$ ), except for $\left(c_{1}, c_{2}\right)$, in two bundles $B_{1}\left(c_{1}\right)$ and $B_{2}\left(c_{1}\right)$ (resp. $B_{1}\left(c_{2}\right)$ and $\left.B_{2}\left(c_{2}\right)\right) . B_{1}\left(c_{1}\right)$ is made up of the edges starting from $\left(c_{1}, b_{m}\right)$ until, but not including, $\left(c_{1}, c_{2}\right)$ in the clockwise order of the edges incident to $c_{1}$. $B_{2}\left(c_{1}\right)$ is made up of the edges starting from $\left(c_{1}, c_{2}\right)$ until, but not including, $\left(c_{1}, b_{m}\right)$ in the clockwise order of the edges incident to $c_{1}$. The other two bundles $B_{1}\left(c_{2}\right)$ and $B_{2}\left(c_{2}\right)$ are defined analogously. $P$ is divided into three subpaths, $P_{1}=$ $\left(c_{1}, a_{l}, a_{l-1}, \ldots, a_{2}, a_{1}\right), P_{2}=\left(c_{1}, b_{1}, b_{2}, \ldots, b_{m}, c_{2}\right)$, and $P_{3}=\left(c_{2}, d_{1}, d_{2}, \ldots, d_{p}\right)$.

Draw $\left(c_{1}, c_{2}\right)$ as an horizontal segment, with $c_{1}$ on the left. $B_{1}\left(c_{1}\right)$ and $B_{2}\left(c_{1}\right)$ $\left(B_{1}\left(c_{2}\right)\right.$ and $\left.B_{2}\left(c_{2}\right)\right)$ are drawn inside wedges centered at $c_{1}$ (at $\left.c_{2}\right)$ and directed rightward (leftward), with $B_{1}\left(c_{1}\right)$ above $\left(c_{1}, c_{2}\right)$ and $B_{2}\left(c_{1}\right)$ below $\left(c_{1}, c_{2}\right)$ (with $B_{1}\left(c_{2}\right)$ above $\left(c_{2}, c_{1}\right)$ and $B_{2}\left(c_{2}\right)$ below $\left.\left(c_{2}, c_{1}\right)\right)$. Such wedges are disjoint and they share an interval $\left[x_{1}, x_{2}\right]$ of the $x$-axis, where $\left[x_{1}, x_{2}\right]$ is a sub-interval of the $x$-extension of the edge $\left(c_{1}, c_{2}\right)$. Draw each edge inside the wedge of its bundle, respecting $\mathcal{E}$ and so that the following rules are observed: the $x$-coordinate of a vertex $b_{i}$ is greater than the one of a vertex $a_{j}$, with $1 \leq i \leq m$ and $1 \leq j \leq l$; the $x$-coordinate of a vertex $d_{k}$ is greater than the one of a vertex $b_{i}$, with $1 \leq k \leq n$ and $1 \leq i \leq m$; the vertices of $P_{1}$, of $P_{2}$, and of $P_{3}$ have increasing, increasing, and decreasing $x$-coordinates, respectively. Each vertex has an $x$-coordinate in the open interval $\left(x_{1}, x_{2}\right)$. Edge $\left(c_{1}, b_{m}\right)\left(\left(c_{2}, b_{1}\right)\right)$ of $D$ is drawn so high (so low) that edge $\left(c_{2}, b_{m}\right)\left(\left(c_{1}, b_{1}\right)\right)$ of $P$ does not create crossings with other edges of the path. The drawing of $D$ is planar since the edges of $D$ are drawn inside disjoint regions of the plane. The absence of crossings in the drawing of $P$ follows from (1) the planarity of the drawings of its subpaths, which in turn follows from the strictly increasing or decreasing $x$-coordinate of its vertices; and (2) from the fact that the subpaths occupy disjoint regions, except for edges $\left(c_{1}, b_{1}\right)$ and $\left(c_{2}, b_{m}\right)$ which do not create crossings, as already discussed. Thus, we have:

Theorem 3. An n-vertex double-star and an n-vertex path not sharing edges admit a geometric simultaneous embedding in which the double-star has a fixed prescribed embedding. 


\subsection{Simultaneous Drawing of Two Caterpillars with Fixed Embedding}

Insisting on a fixed embedding when simultaneously embedding planar graphs is a very restrictive requirement as shown by the following theorem:

Theorem 4. It is not always possible to find a geometric simultaneous embedding for two caterpillars with fixed embeddings.

Proof: Let $C_{1}$ and $C_{2}$ be the two caterpillars with fixed embeddings $\mathcal{E}_{1}$ and $\mathcal{E}_{2}$ and a bijective mapping $\gamma(x)=x$ between their vertices; see Fig. 3(a-b). We now show that there does not exist a geometric simultaneous embedding of $C_{1}$ and $C_{2}$ in which $C_{1}$ and $C_{2}$ respect $\mathcal{E}_{1}$ and $\mathcal{E}_{2}$, respectively.

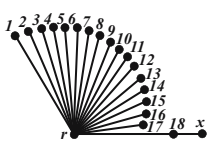

(a)

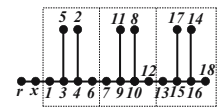

(b)

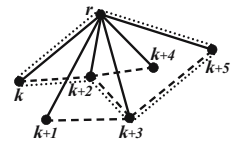

(c)

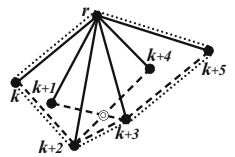

(d)

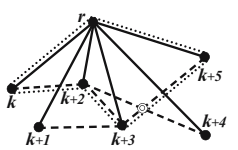

(e)

Fig. 3. (a)-(b) Caterpillars $C_{1}$ and $C_{2}$; (c)-(e) Illustrations for the proof of Theorem 4

Construct a straight-line drawing $\Gamma_{1}$ of $C_{1}$. The embedding $\mathcal{E}_{1}$ of $C_{1}$ forces the vertices $1,2, \ldots, 18$ to appear in this order around $r$ in $\Gamma_{1}$. Consider the subtrees of $C_{1}$ induced by the vertices $r, 1,2, \ldots, 6$, by the vertices $r, 7,8, \ldots, 12$, and by the vertices $r, 13,14, \ldots, 18$. Since such subtrees appear consecutively around $r$, then at least one of them must be drawn in a wedge rooted at $r$ and with angle less than $\pi$. Let $C_{S}$ be such a subtree and let $k, k+1, \ldots, k+5$ be the vertices of $C_{S}$, with $k=1,7$ or 13 . Without loss of generality, let $r$ be the uppermost point of this wedge. It follows that $C_{S}$ must be drawn downward. Denote by $P$ the polygon composed of the edges $(r, k)$ and $(r, k+5)$ of $C_{1}$ and of the edges $(k, k+2),(k+2, k+3)$, and $(k+3, k+5)$ of $C_{2}$. Note that vertices $k+1$ and $k+4$ must be either both inside or both outside $P$. In fact, placing one of these vertices inside and the other outside $P$ is not consistent with the embedding constraints of $\mathcal{E}_{2}$; see Fig. 3 (c). If both vertices $k+1$ and $k+4$ are placed inside $P$, then the embedding constraints of $\mathcal{E}_{1}$ and $\mathcal{E}_{2}$ and the upwardness of $C_{S}$ imply that edge $(k+2, k+4)$ must cut edge $(r, k+3)$ and that edge $(k+1, k+3)$ must cut edge $(r, k+2)$. It follows that there is an intersection between edges $(k+2, k+4)$ and $(k+1, k+3)$, both belonging to $C_{S}$; see Fig. 3(d). Similarly, if both vertices $k+1$ and $k+4$ are placed outside $P$, then by the embedding constraints of $\mathcal{E}_{1}$ and $\mathcal{E}_{2}$ vertex $k+2$ is placed inside the polygon formed by the edges $(r, k+1),(r, k+5)$ of $C_{1}$ and by the edges $(k+1, k+3),(k+3, k+5)$ of $C_{2}$. Hence, edge $(k+2, k+4)$ cuts such a polygon either in edge $(k+1, k+3)$ or in edge $(k+3, k+5)$; see Fig. 3 (e) and this concludes the proof. 


\subsection{Simultaneous Drawing of Outerplanar Graphs and Paths with Fixed Embedding}

Let $O^{*}$ be the outerplanar graph on vertices $v_{1}, v_{2}, \ldots, v_{7}$ shown in Fig. 4(a) and $\mathcal{E}^{*}$ be the embedding of $O^{*}$ shown in Fig. 4 (b). Let $F^{*}$ be the face of $\mathcal{E}^{*}$ with incident vertices $v_{1}, v_{3}$, and $v_{7}$ and let $P^{*}$ be the path $\left(v_{1}, v_{2}, v_{3}, v_{4}, v_{5}, v_{6}, v_{7}\right)$.

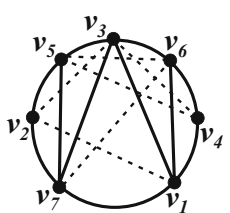

(a)

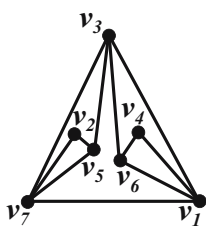

(b)

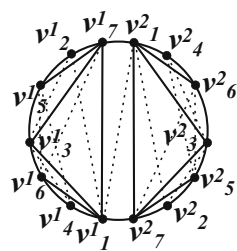

(c)

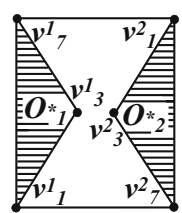

(d)

Fig. 4. (a) Outerplanar graph $O^{*}$, drawn with solid edges, and path $P^{*}$, drawn with dashed edges. (b) Embedding $\mathcal{E}^{*}$ of $O^{*}$. (c) Outerplanar graph $O$, drawn with solid edges, and path $P$, drawn with dashed edges. (d) Embedding $\mathcal{E}$ of $O$.

Lemma 2. There does not exist a geometric simultaneous embedding of $O^{*}$ and $P^{*}$ in which the embedding of $O^{*}$ is $\mathcal{E}^{*}$ and the external face of $O^{*}$ is $F^{*}$.

Theorem 5. There exist an outerplanar graph $O$, an embedding $\mathcal{E}$ of $O$, a path $P$, and a mapping between their vertices such that: (i) $O$ and $P$ do not share edges, and (ii) $O$ and $P$ have no geometric simultaneous embedding.

Proof: Let $O_{1}^{*}$ and $O_{2}^{*}$ be two copies of the outerplanar graph $O^{*}$ defined above. Denote by $v_{i}^{j}$, with $j=1,2$ and $i=1, \ldots, 7$, the vertex of $O_{j}^{*}$ that corresponds to vertex $v_{i}$ of $O^{*}$ in $O$. Let $\mathcal{E}_{1}^{*}$ and $\mathcal{E}_{2}^{*}$ be the embeddings of $O_{1}^{*}$ and $\mathrm{O}_{2}^{*}$ corresponding to the embedding $\mathcal{E}^{*}$ of $O^{*}$. Let $O$ be the graph composed of $O_{1}^{*}$, of $O_{2}^{*}$, and of edges $\left(v_{7}^{1}, v_{1}^{2}\right),\left(v_{1}^{1}, v_{7}^{2}\right)$; see Fig. 4 4 (c). Let the embedding $\mathcal{E}$ for $O$ be defined as follows: (i) each vertex of $O_{1}^{*}$ (of $O_{2}^{*}$ ) but for $v_{1}^{1}$ and $v_{7}^{1}$ (but for $v_{1}^{2}$ and $v_{7}^{2}$ ) has the same adjacency list as in $\mathcal{E}_{1}^{*}$ (in $\mathcal{E}_{2}^{*}$ ); (ii) the adjacency lists of the remaining vertices are as follows: $v_{1}^{1} \rightarrow\left(v_{7}^{1}, v_{6}^{1}, v_{4}^{1}, v_{3}^{1}, v_{7}^{2}\right)$, $v_{7}^{1} \rightarrow\left(v_{1}^{2}, v_{3}^{1}, v_{2}^{1}, v_{5}^{1}, v_{1}^{1}\right), v_{1}^{2} \rightarrow\left(v_{7}^{2}, v_{6}^{2}, v_{4}^{2}, v_{3}^{2}, v_{7}^{1}\right), v_{7}^{2} \rightarrow\left(v_{1}^{1}, v_{3}^{2}, v_{2}^{2}, v_{5}^{2}, v_{1}^{2}\right)$. Let $P$ be the path $\left(v_{7}^{1}, v_{6}^{1}, v_{5}^{1}, v_{4}^{1}, v_{3}^{1}, v_{2}^{1}, v_{1}^{1}, v_{1}^{2}, v_{2}^{2}, v_{3}^{2}, v_{4}^{2}, v_{5}^{2}, v_{6}^{2}, v_{7}^{2}\right) . O$ and $P$ do not share edges, and the subpaths of $P$ induced by the vertices of $O_{1}^{*}\left(O_{2}^{*}\right)$ play for $O_{1}^{*}\left(O_{2}^{*}\right)$ the same role that path $P^{*}$ plays for graph $O^{*}$ in Lemma 2 .

Let $F_{1}^{*}$ and $F_{2}^{*}$ denote cycles $\left(v_{1}^{1}, v_{3}^{1}, v_{7}^{1}\right)$ and $\left(v_{1}^{2}, v_{3}^{2}, v_{7}^{2}\right)$, respectively. These cycles are faces of $O_{1}^{*}$ and $O_{2}^{*}$. We now show that every plane drawing $\Gamma_{\mathcal{E}}$ of $O$ with embedding $\mathcal{E}$ determines a non-planar drawing of $P$. Consider the embed$\operatorname{ding} \mathcal{E}_{O}$ of $O$ obtained by choosing $\left(v_{1}^{1}, v_{7}^{1}, v_{1}^{2}, v_{7}^{2}\right)$ as external face; see Fig. 4(d). Choosing any face external to $F_{1}^{*}\left(F_{2}^{*}\right)$ in $\mathcal{E}_{O}$ as external face of $\Gamma_{\mathcal{E}}$ leaves $O_{1}^{*}$ $\left(O_{2}^{*}\right)$ embedded with external face $F_{1}^{*}\left(F_{2}^{*}\right)$. Hence, we can apply Lemma 2 and conclude that there is no simultaneous embedding of $O$ and $P$. 


\section{$5 \quad$ Near-Simultaneous Embedding}

In this section we study the variation of geometric simultaneous embedding in which vertices representing the same entity in different graphs can be placed in different points in different drawings. However, in order to preserve the viewer's "mental map" corresponding vertices should be placed as close as possible. This turns out to be impossible for general planar graphs, as the first lemma of this section shows. First, define the displacement of a vertex $v$ between two drawings $\Gamma_{1}$ and $\Gamma_{2}$ as the distance between the location of $v$ in $\Gamma_{1}$ and the location of $v$ in $\Gamma_{2}$. Second, we show that there exist two $n$-vertex planar graphs $G_{1}$ and $G_{2}$ with a bijection $\gamma$ between their vertices such that for any two planar straight-line grid drawings $\Gamma_{1}$ and $\Gamma_{2}$ of $G_{1}$ and $G_{2}$, respectively, there exists a vertex $v$ that has a displacement $\Omega(n)$ between $\Gamma_{1}$ and $\Gamma_{2}$.

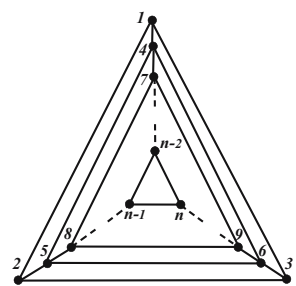

(a)

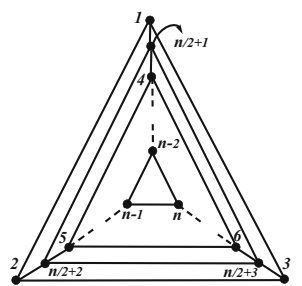

(b)

Fig. 5. (a) Nested triangle graph $G_{1}$; (b) Nested triangle graph $G_{2}$

Let $G_{1}$ and $G_{2}$ be two $n$-vertex nested triangle graphs; see Fig. 5. A nested triangle graph $G$ is a triconnected planar graph with a triangular face $F(G)$ such that removing the vertices of $F(G)$ and their incident edges leaves a smaller nested triangle graph or an empty vertex set. Suppose the mapping $\gamma\left(v_{1}\right)=v_{2}$ between vertices $v_{1} \in V\left(G_{1}\right)$ and vertices $v_{2} \in V\left(G_{2}\right)$ is the one shown in Fig. 5 and defined by the following procedure: embed $G_{1}$ and $G_{2}$ with external faces $F\left(G_{1}\right)$ and $F\left(G_{2}\right)$, respectively. Starting from $G_{1}\left(G_{2}\right)$, for $i=1, \ldots, n / 3$, remove from the current graph the three vertices of the external face and label them $3 i-2,3 i-1$, and $3 i(3(i+1) / 2-2,3(i+1) / 2-1$, and $3(i+1) / 2$ if $i$ is odd, or $(n+3 i) / 2-2,(n+3 i) / 2-1$, and $(n+3 i) / 2$ if $i$ is even). Then, for any two planar straight-line grid drawings $\Gamma_{1}$ of $G_{1}$ and $\Gamma_{2}$ of $G_{2}$ and $G_{2}$, we have:

Lemma 3. There exists a vertex representing the same entity in $G_{1}$ and $G_{2}$ that has displacement $\Omega(n)$ between $\Gamma_{1}$ and $\Gamma_{2}$.

The lower bound in Lemma 3 is easily matched by an upper bound obtained by independently drawing each planar graph in $O(n) \times O(n)$ area: Each vertex is displaced by at most the length of the diagonal of the drawing's bounding box. Clearly, such a diagonal has length $O(n)$.

The above result shows that we cannot hope to guarantee near-simultaneous embeddings for arbitrary pairs of planar graphs. It is possible, however, that for graphs that are "similar", near-simultaneous embeddings might exist. Similarity 
between graphs could be defined and regarded in several different ways, by minding both the combinatorial structure of the graphs and the mapping between the vertices of the graphs. With this in mind, in the following we look for nearsimultaneous embeddings of similar paths and similar trees.

\subsection{Near-Simultaneous Drawings of Similar Paths}

Recall that two paths always have a geometric simultaneous embedding, while three of them might not have one 1. Therefore, in order to represent a sequence of paths using a sequence of planar drawings, vertices that are in correspondence under the mapping must be displaced from one drawing to the next.

Observing that a path induces an ordering of the vertices, call two $n$-vertex paths $P_{1}$ and $P_{2}$ with orderings $\pi_{1}$ and $\pi_{2}$ of their vertices and with a bijective mapping $\gamma$ between their vertices $k$-similar if for each vertex $v_{1} \in P_{1}$ the position of $v_{1}$ in $\pi_{1}$ differs by at most $k$ positions from the one of $v_{2}=\gamma\left(v_{1}\right)$ in $\pi_{2}$. Drawing the paths as horizontal polygonal lines with uniform horizontal distances between adjacent vertices gives a near-simultaneous drawing. As any vertex $v_{i}$ if $P_{1}$ occurs within $k$ positions in $P_{2}$ (compared with its position in $P_{1}$ ) then the extent of the displacement of the vertex from one drawing to the next is limited by exactly $k$ units. More generally, this idea can be summarized as follows:

Theorem 6. A sequence of $n$-vertex paths $P_{0}, P_{1}, \ldots, P_{m}$, where each two consecutive paths are $k$-similar, can be drawn so that the displacement of any vertex in a pair of paths that are consecutive in the sequence is at most $k$.

\subsection{Near-Simultaneous Drawings of Similar Trees}

Generalizing the idea of $k$-similarity to trees, call two rooted arbitrarily ordered trees $T_{1}$ and $T_{2}$ with vertex sets $V_{1}$ and $V_{2}$ and with bijective mapping $\gamma$ between their vertices, $k$-similar if: (i) The depths of any vertex $v_{1} \in V_{1}$ and of its corresponding vertex $\gamma\left(v_{1}\right) \in V_{2}$ differ by at most $k$; (ii) The positions of any two corresponding vertices in any pre-established traversal of the tree among pre-, in-, post-order, or breadth-first-search traversal differ by at most $k$.

Given two trees $T_{1}$ and $T_{2}$ that are $k$-similar with respect to a pre-established traversal order $\pi$, we can draw each of $T_{1}$ and $T_{2}$ as follows: (1) Assign to each vertex $v_{i}$ its position $\pi\left(v_{i}\right)$ as an $x$-coordinate; (2) Assign to each vertex $v_{i}$ its depth as a $y$-coordinate.

Such an algorithm produces layouts that are planar and layered. A drawing is layered if (i) each vertex is assigned to a layer, (ii) for each layer an order of its vertices is specified, and (iii) there are only edges joining vertices on consecutive layers. Since subsequent trees are $k$-similar, the depth of any vertex and its position in a tree traversal changes only by $k$ in two consecutive trees; hence, we have that the displacement of a vertex representing the same entity in different drawings is at most $\sqrt{k^{2}+k^{2}}=k \sqrt{2}$. More generally, we have the following:

Theorem 7. A sequence of $n$-vertex trees $T_{0}, T_{1}, \ldots, T_{m}$, where each two consecutive trees are $k$-similar, can be drawn such that the displacement of any vertex in a pair of trees that are consecutive in the sequence is at most $k \sqrt{2}$. 
Observe that an analogous definition of similarity between two graphs and the same layout algorithm work more generally for level planar graphs [910] (and hence for outerplanar graphs). Finally, the area requirement of the drawings produced by the described algorithm is worst-case quadratic in the number of vertices of a tree (or of a level planar graph).

\section{Conclusions}

In this paper we have considered some variations of the well-known problem of embedding graphs simultaneously.

Concerning the geometric simultaneous embedding without common edges, we provided a negative result that seems to show that the geometric simultaneous embedding is not more powerful by assuming the edge sets of the input graphs to be disjoint. Further, we believe that there exist two trees not sharing common edges that do not admit a geometric simultaneous embedding. This would extend the result in [8] where two trees that do not admit a simultaneous embedding and that do share edges are shown. Consider two isomorphic rooted trees $T_{1}(h, k)$ and $T_{2}(h, k)$ a mapping $\gamma$ between their vertices defined as follows (see Fig. 6): (i) the root of $T_{1}(h, k)$ (of $T_{2}(h, k)$ ) has $k$ children; (ii) each vertex of $T_{1}(h, k)$ (of $\left.T_{2}(h, k)\right)$ at distance $i$ from the root, with $1 \leq i<h$, has a number of children one less than the number of vertices at distance $i$ from the root in $T_{1}(h, k)$ (in $T_{2}(h, k)$ ); (iii) one vertex of $T_{1}(h, k)$ (of $\left.T_{2}(h, k)\right)$ at distance $h$ from the root has one child; (iv) each child of the root of $T_{1}(h, k)$ is mapped to a distinct child of the root of $T_{2}(h, k) ;(\mathrm{v})$ for each pair of vertices $v_{1}$ of $T_{1}(h, k)$ and $v_{2}$ of $T_{2}(h, k)$ that are at distance $i$ from the root of their own tree and that are such that $v_{2} \neq \gamma\left(v_{1}\right)$, there exists a child of $v_{1}$ that is mapped to a child of $v_{2}$; (vi) the only vertex of $T_{1}(h, k)$ (of $T_{2}(h, k)$ ) that is at distance $h+1$ from the root is mapped to the root of $T_{2}(h, k)$ (to the root of $\left.T_{1}(h, k)\right)$.

Conjecture 1. For sufficiently large $h$ and $k, T_{1}(h, k)$ and $T_{2}(h, k)$ do not admit a geometric simultaneous embedding with mapping $\gamma$ between their vertices.

For the problem of drawing graphs simultaneously with fixed embedding, we provided more negative results than in the usual setting for geometric simultaneous

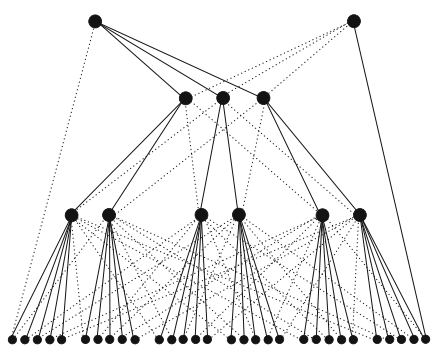

Fig. 6. Trees $T_{1}(3,3)$ and $T_{2}(3,3)$ with the mapping $\gamma$ between their vertices. $T_{1}(3,3)$ has solid edges and $T_{2}(3,3)$ has dashed edges. 
embedding, while providing only two positive results partially covering the ones already known for geometric simultaneous embedding. We believe that understanding the possibility of obtaining a simultaneous embedding of a tree and a path in which the tree has a fixed embedding could be useful for the same problem in the non-fixed embedding setting.

Even in the more relaxed near-simultaneous setting, we have shown that without assuming a similarity in the sequence of graphs to be drawn, it is difficult to limit the displacement of a vertex from a drawing to the next. We have shown that for paths, for trees, and for level planar graphs there exist reasonable similarity measures that allow us to obtain near-simultaneous drawings. However, in the case of general planar graphs it is not yet clear what kind of similarity metric can be defined and how well can such graphs be drawn.

\section{Acknowledgments}

Thanks to Markus Geyer for useful discussions.

\section{References}

1. Braß, P., Cenek, E., Duncan, C.A., Efrat, A., Erten, C., Ismailescu, D., Kobourov, S.G., Lubiw, A., Mitchell, J.S.B.: On simultaneous planar graph embeddings. Comput. Geom. 36(2), 117-130 (2007)

2. Collberg, C., Kobourov, S.G., Nagra, J., Pitts, J., Wampler, K.: A system for graphbased visualization of the evolution of software. In: SoftVis, pp. 377-386 (2003)

3. Di Battista, G., Eades, P., Tamassia, R., Tollis, I.G.: Graph Drawing. Prentice Hall, Upper Saddle River, NJ (1999)

4. Di Giacomo, E., Liotta, G.: A note on simultaneous embedding of planar graphs. In: EWCG, pp. 207-210 (2005)

5. Erten, C., Kobourov, S.G.: Simultaneous embedding of planar graphs with few bends. In: Pach, J. (ed.) GD 2004. LNCS, vol. 3383, pp. 195-205. Springer, Heidelberg (2005)

6. Frati, F.: Embedding graphs simultaneously with fixed edges. In: Kaufmann, M., Wagner, D. (eds.) GD 2006. LNCS, vol. 4372, pp. 108-113. Springer, Heidelberg (2007)

7. Frati, F., Kaufmann, M., Kobourov, S.: Constrained simultaneous and nearsimultaneous embeddings. Tech. Report RT-DIA-120-2007, University of Roma Tre (2007), http://dipartimento.dia.uniroma3.it/ricerca/rapporti/rt/2007-120.pdf

8. Geyer, M., Kaufmann, M., Vrto, I.: Two trees which are self-intersecting when drawn simultaneously. In: Healy, P., Nikolov, N.S. (eds.) GD 2005. LNCS, vol. 3843, pp. 201-210. Springer, Heidelberg (2006)

9. Healy, P., Kuusik, A., Leipert, S.: A characterization of level planar graphs. Discr. Math. 280(1-3), 51-63 (2004)

10. Jünger, M., Leipert, S.: Level planar embedding in linear time. Jour. of Graph Alg. and Appl. 6(1), 67-113 (2002)

11. Kaufmann, M., Wagner, D. (eds.): Drawing Graphs. LNCS, vol. 2025. Springer, Heidelberg (2001) 